\title{
DYNAMO THEORY OF THE SUN'S GENERAL MAGNETIC FIELD \\ ON THE BASIS OF A MEAN-FIELD MAGNETOHYDRODYNAMICS
}

\author{
F. KRAUSE and K.-H. RÄDLER \\ Zentralinstitut für Astrophysik, Potsdam, Germany
}

\begin{abstract}
An outline of the mean-field magnetohydrodynamics suggested and developed by $M$. Steenbeck and the authors and its application to the dynamo theory of the solar cycle is presented. Four basic requirements are formulated which have to be satisfied by any dynamo model which claims to explain the solar cycle. The models investigated allow conclusions about the differential rotation. In this connection Leighton's work is criticized.
\end{abstract}

\section{Basic Ideas}

The general magnetic field of the Sun is observed to be an alternating field, therefore the possibility of a relic field is almost completely excluded. In this way the idea that the Sun is a self-excited dynamo seems to be the only one promising success for the explanation of the magnetic field on the basis of classical physics.

According to our opinion any dynamo model for the Sun has to satisfy the following four requirements:

(1) The dynamo action must be provided by motions, which can be expected at the Sun;

(2) the dynamo excitation must occur for arbitrarily small seed fields;

(3) the dynamo must be an alternating field dynamo;

(4) the excited field must be antisymmetric with respect to the equatorial plane.

According to requirement (1) one has to take velocity fields, which correspond to the observations or are derived from the Navier-Stokes equation under appropriate conditions. Requirement (2) is formulated according to the conception that without dynamo action only insignificantly small fields can exist at the Sun. A first consequence of (2) is that we are allowed to neglect the Lorentz force in excitation. A second consequence of this requirement is that the feedback mechanism proposed by Babcock (1961) cannot be accepted. Babcock suggests that the desired production of poloidal field from the toroidal field is given by the systematic tilt of sunspot pairs. This observed tilt clearly gives a production of a poloidal field, however, this mechanism does not work until the field strength is greater than a certain critical value. Hence a dynamo using this mechanism cannot work at arbitrarily small fields. Leighton (1969) allowed this feedback mechanism to work also for arbitrarily small fields, but we will return to this paper later.

Requirement (3) expresses the observed fact that the Sun's general magnetic field is an alternating one with a period of $22 \mathrm{yr}$, and requirement (4) that the field shows the opposite orientation regarding space points situated symmetric to the equatorial plane. Whereas requirement (3) expresses the change of the polarity from one cycle to 
the following, requirement (4) expresses that the polarity also changes from one hemisphere of the Sun to the other. Requirement (4), for instance, includes the observation that the preceding spots of one hemisphere have the opposite polarity of the preceding spots on the other hemisphere.

The formulation of these four basic requirements is rather general. Obviously one can formulate more detailed requirements, because more detailed results are furnished by observations. The main reason for choosing these four basic requirements is that they can be satisfied by a linear theory as is shown in this paper. Such details of the solar field as the butterfly diagram are apparently strongly influenced by non-linear effects (action of the Lorentz force on motion). Therefore it is of less value to require from a linear theory very accurately fitted models. It is clear how to include the non-linear effects in this theory but then great mathematical difficulties will arise. That will be a matter of work to be done in future.

\section{Mean-Field Magnetohydrodynamics}

As is well known there exists no solution of the dynamo problem in which both magnetic and velocity fields have a simple structure. One is tempted to draw attention to the turbulence. The plasma of the convection zone shows turbulent motions and there are some hints that they are important for the dynamo action. In this connection a mean-field magnetohydrodynamics seems to be the appropriate theory, which was suggested and developed in earlier papers (Steenbeck et al., 1966; Rädler, 1968; Krause, 1968, 1969; and Rädler, 1969). We give only an outline of the basic ideas in the present paper*.

The basic equations of magnetohydrodynamics are:

Maxwell's equations

$$
\operatorname{curl} \mathbf{E}=-\dot{\mathbf{B}}, \quad \operatorname{curl} \mathbf{H}=\mathbf{j}, \quad \operatorname{div} \mathbf{B}=0,
$$

the constitutive equations

$$
\mathbf{B}=\mu \mathbf{H}, \quad \mathbf{j}=\sigma(\mathbf{E}+\mathbf{v} \times \mathbf{B}),
$$

the Navier-Stokes equation

$$
\varrho(\partial \mathbf{v} / \partial t)+(\mathbf{v} \cdot \operatorname{grad}) \mathbf{v}=-\operatorname{grad} p+\mathbf{j} \times \mathbf{B}+\mathbf{F}
$$

and further equations which, as well as the forces $\mathbf{F}$ in the Navier-Stokes equation, need not be given explicitly here. We use the notations: $\mathbf{E}$ electric field strength, $\mathbf{B}$ magnetic induction, $\mathbf{H}$ magnetic field strength, $\mathbf{j}$ current density, $\mathbf{v}$ velocity field, $p$ pressure, $\varrho$ density.

* An extended representation of the mean-field magnetohydrodynamics will appear soon in Rompe and Steenbeck, Handbuch der Plasmaphysik und der Gaselektronik, Band II, Akademie-Verlag Berlin. 
We get the basic equations for the mean fields by averaging the foregoing equations (1), (2), (3). Averaged quantities are denoted by a bar and fluctuations by a dash. We have

$$
\begin{aligned}
& \operatorname{curl} \overline{\mathbf{E}}=-\dot{\overline{\mathbf{B}}}, \quad \operatorname{curl} \overline{\mathbf{H}}=\overline{\mathbf{j}}, \quad \operatorname{div} \overline{\mathbf{B}}=0 \\
& \overline{\mathbf{B}}=\mu \overline{\mathbf{H}}, \quad \overline{\mathbf{j}}=\sigma\left(\overline{\mathbf{E}}+\overline{\mathbf{v}} \times \overline{\mathbf{B}}+\overline{\left.\mathbf{v}^{\prime} \times \mathbf{B}^{\prime}\right)}\right. \\
& \varrho\left(\frac{\partial \overline{\mathbf{v}}}{\partial t}+(\overline{\mathbf{v}} \cdot \operatorname{grad}) \overline{\mathbf{v}}\right)=-\operatorname{grad} \bar{p}+\overline{\mathbf{j}} \times \overline{\mathbf{B}}+\overline{\mathbf{F}}+\overline{\mathbf{j}^{\prime} \times \mathbf{B}^{\prime}}-\overline{\varrho\left(\mathbf{v}^{\prime} \cdot \operatorname{grad}\right) \mathbf{v}^{\prime}}
\end{aligned}
$$

Maxwell's equations are valid for the mean fields as well as for the original fields. This is a consequence of their linearity. But the non-linear terms involved in Ohm's law and in the Navier-Stokes equation provide for new physical quantities like the electromotive force $\overline{\mathbf{v}^{\prime} \times \mathbf{B}^{\prime}}$ and the ponderomotive forces $\overline{\mathbf{j}^{\prime} \times \mathbf{B}^{\prime}}$ and $-\overline{\varrho\left(\mathbf{v}^{\prime} \cdot \operatorname{grad}\right) \mathbf{v}^{\prime}}$.

In order to get a closed system of differential equations again one has to express the additional terms $\overline{\mathbf{v}^{\prime} \times \mathbf{B}^{\prime}},-\overline{\varrho\left(\mathbf{v}^{\prime} \cdot \operatorname{grad}\right) \mathbf{v}^{\prime}}, \overline{\mathbf{j}^{\prime} \times \mathbf{B}^{\prime}}$ by the mean fields. The analysis of this problem is developed to some extent for the electromotive force $\overline{\mathbf{v}^{\prime} \times \mathbf{B}^{\prime}}$ (Steenbeck et al., 1966; Rädler, 1968; and Krause, 1969). The other two terms can be treated in an analogous manner (Krause, 1969), but more detailed work is needed.

For a treatment of the dynamo problem according to our requirements (1)-(4) we need to be concerned with the emf $\overline{\mathbf{v}^{\prime} \times \mathbf{B}^{\prime}}$ only. The explicit expression of this emf $\overline{\mathbf{v}^{\prime} \times \mathbf{B}^{\prime}}$ will depend on the structure of the turbulence. A turbulence like that performed by the plasma of the convection zone undergoes in particular the influence of some vector quantities, like the gravity force, the density gradient, the temperature gradient and finally the rotational motion represented by the pseudo-vector $\omega$ of the angular velocity. The first three vector quantities are parallel, parallel to the radial direction, and their influence is subsumed by the general property of the turbulence that the radial direction is a preferred direction, a direction of anisotropy. We denote it by $\mathbf{g}$. In addition the direction parallel to the axis of rotation is a preferred direction, but this is represented by the pseudo-vector $\omega$. Any average vector field can be a combination of these two vectors $\mathbf{g}$ and $\boldsymbol{\omega}$ and in addition the mean magnetic field $\overline{\mathbf{B}}$ only. It may be noticed that $\overline{\mathbf{B}}$ is also a pseudo-vector, whereas $\overline{\mathbf{v}^{\prime} \times \mathbf{B}^{\prime}}$ is a vector.

Taking into account the general symmetry laws of physics the emf $\overline{\mathbf{v}^{\prime} \times \mathbf{B}^{\prime}}$ can only be an expression like

$$
\begin{aligned}
\overline{\mathbf{v}^{\prime} \times \mathbf{B}^{\prime}}= & -\alpha_{1}(\mathbf{g} \cdot \omega) \overline{\mathbf{B}}-\alpha_{2}(\mathbf{g} \cdot \overline{\mathbf{B}}) \omega-\alpha_{3}(\omega \cdot \overline{\mathbf{B}}) \mathbf{g} \\
& -\beta \operatorname{curl} \overline{\mathbf{B}}-\beta_{1} \omega \times \operatorname{curl} \overline{\mathbf{B}}-\beta_{2} \operatorname{grad}(\omega \cdot \overline{\mathbf{B}})-\gamma \mathbf{g} \times \overline{\mathbf{B}},
\end{aligned}
$$

where $\alpha_{1}, \alpha_{2}, \alpha_{3}, \ldots, \gamma$ are scalars.

The most important result we find is an emf $\alpha \overline{\mathbf{B}}$ parallel to the mean magnetic field. We have now introduced the pseudo-scalar according to

$$
\alpha=-\alpha_{1} \mathbf{g} \cdot \omega
$$


This effect of establishing an emf parallel to the magnetic field is a quite natural effect appearing in turbulence in rotating systems, although it is quite unknown in common electrodynamics. We call it the ' $\alpha$-effect'. This effect can be obtained from general physical arguments as shown here. In order to determine the value of the scalar $\alpha_{1}$ more detailed calculation is needed.

There is a close relationship between $\alpha$ and the appearance of helical motions in a conducting fluid. $\alpha$ is positive if there are motions according to a lefthanded screw being more probable than motions according to a righthanded screw. $\alpha$ is negative in the opposite case. According to Moffatt (1970) a turbulence of this kind is called 'turbulence with helicity'.* A characteristic property of a turbulence with helicity is that the average $\overline{\mathbf{v}^{\prime} \cdot \text { curl }^{\prime}}$ is not equal to zero. The helicity of the turbulence within the convection zone is furnished by the action of the Coriolis forces. As a consequence of (8) a dependence proportional to $\cos \theta$ is expected, where $\theta$ is the colatitude.

A calculation of $\alpha$ was made by a perturbation method (Steenbeck et al., 1966; Krause, 1968). An inhomogeneous and consequently anisotropic turbulence was perturbed by a rotational motion. The following result was obtained (Krause, 1968)

$$
\alpha=\alpha_{0} \cos \theta, \quad \alpha_{0}=\tau_{\text {cor }}^{2} v_{\text {eff }}^{2} \omega / L .
$$

$\tau_{\text {cor }}$ denotes the correlation time, $v_{\text {eff }}$ the rms value of the velocity and $L$ the scaleheight. With the data observed at the surface of the Sun we have

$$
\alpha_{0} \approx 0.3 \mathrm{~m} / \mathrm{s} \text {. }
$$

The $\alpha$-effect is of interest for dynamo theory (Krause, 1968; Moffatt, 1970; Steenbeck and Krause, 1966, 1968; Vainstein, 1970; and Fitremann and Frisch, 1969). This is so because toroidal currents are caused by toroidal magnetic fields by means of the $\alpha$-effect. In this case one gets a closed circuit: Toroidal magnetic fields will be produced from poloidal magnetic fields by the differential rotation, and poloidal magnetic fields will be produced from toroidal magnetic fields by the action of the $\alpha$-effect.

\section{The Dynamo Models}

For the Sun there arises a certain difficulty which is related to the observed fact that the Sun has an alternating magnetic field with the well-known period of $22 \mathrm{yr}$. The situation is depicted in Figure 3 schematically.

The field $\mathbf{B}_{\mathrm{tor}}^{(1)}$ is produced from $\mathbf{B}_{\mathrm{pol}}^{(1)}$ by the differential rotation $\mathbf{B}_{\mathrm{pol}}^{(2)}$ with the desired opposite sign appearing because of the toroidal currents caused by the field $\mathbf{B}_{\text {tor }}^{(1)}$ by means of the $\alpha$-effect. However, if the $\alpha$-effect and the differential rotation act at the same place at the same time, the original field $\mathbf{B}_{\mathrm{pol}}^{(1)}$ is weakened immediately by the new field $\mathbf{B}_{\mathrm{pol}}^{(2)}$ and as a result there will be a damped oscillation which dies out during a few periods.

* In German 'Turbulenz mit bevorzugtem Schraubensinn'. 

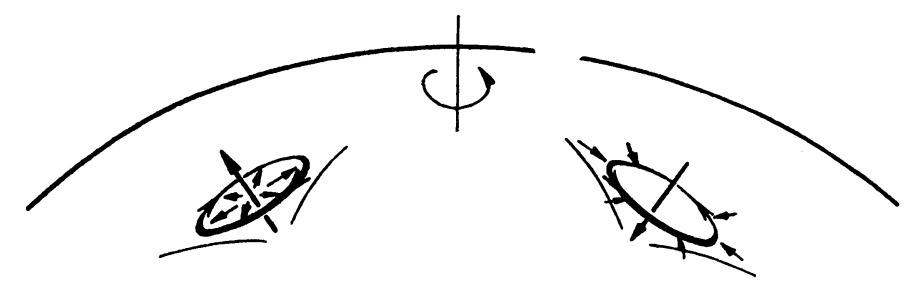

Fig. 1. This figure illustrates the helicity of the convection in the northern hemisphere of the Sun. Rising matter expands because of the density gradient and undergoes the influence of Coriolis forces. A motion according to a lefthanded screw results. For sinking matter both the radial motion and the circulation change sign; hence a motion according to a lefthanded screw appears too.

This behavior does not appear if the two induction mechanisms act at different places (Krause and Steenbeck, 1965). In Figure 4 we have two layers separated by a third layer of thickness $\delta$. In one layer $\operatorname{grad} \omega$, in the other $\alpha$, is not equal to zero. Now we have to regard each field component diffusing through the separating layer. We distinguish the fields at the places where they are induced and where they occur by diffusion, and mark them by the indices $i$ and $d$. If we have $\mathbf{B}_{\mathrm{pol}}^{(d)}$ in the left layer we get $\mathbf{B}_{\text {tor }}^{(1 i)}$ by the action of the differential rotation. Now $\mathbf{B}_{\text {tor }}^{(1 i)}$ diffuses and after a time of about $\mu \sigma \delta^{2}$ we have $\mathbf{B}_{\text {tor }}^{(d)}$ in the right layer, where the field $\mathbf{B}_{\mathrm{pol}}^{(2 i)}$ is generated by the $\alpha$-effect. Now $\mathbf{B}_{\mathrm{pol}}^{(2 i)}$ also diffuses and reaches the left layer, weakening $\mathbf{B}_{\mathrm{pol}}^{(1)}$ and finally changing the sign of it. The distance $\delta$ between both layers determines the frequency $\Omega$; there is some proportionality between $\Omega$ and $1 / \mu \sigma \delta^{2}$, roughly speaking. Explicit numerical treatments of some models proved that this mechanism works (Steenbeck and Krause, 1968). The basic form of this model is depicted in Figure 5.

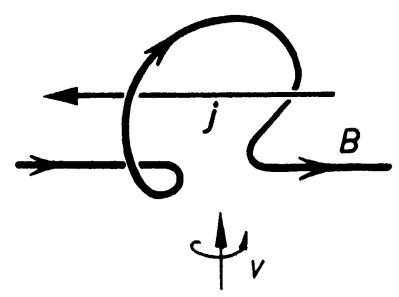

Fig. 2. A magnetic flux line undergoing a helical motion is deformed to a twisted $\Omega$. For a motion according to a righthanded screw, as depicted here, this deformation is accompanied by a current antiparallel to the magnetic field ( $\alpha$ negative). For lefthanded screw motions the accompanying current is parallel ( $\alpha$ positive).

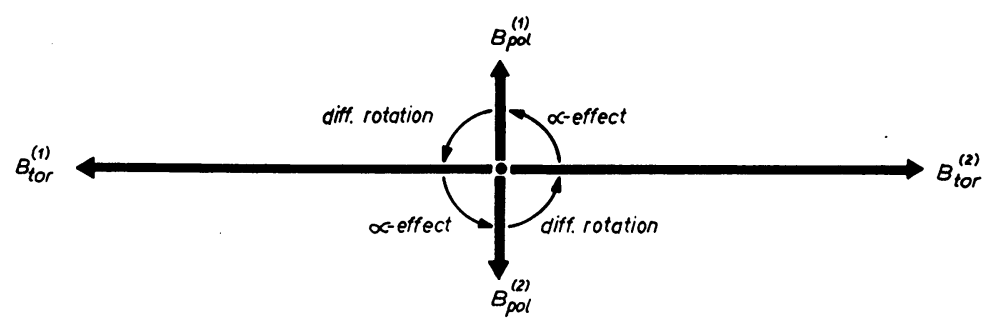

Fig. 3. Schematic representation of a model where both the induction mechanisms act at the same place. The weakening of the original field occurs immediately and damped oscillating field results. 


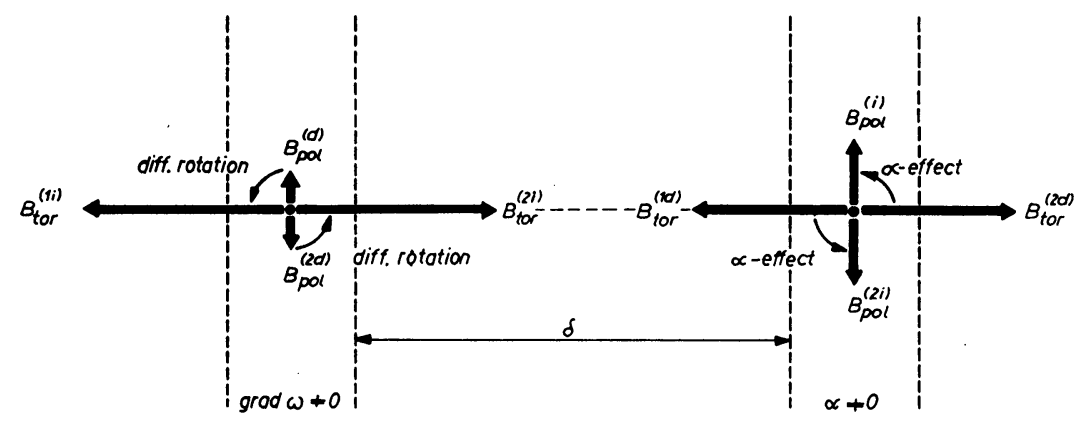

Fig. 4. Schematic representation of a model where different induction mechanisms act at different layers separated by an additional layer. The induced field needs a certain time for diffusing through the separating layer. Hence the weakening of the original field occurs with a certain time retardation.

This model is proved to maintain an oscillating field (Steenbeck and Krause, 1968).

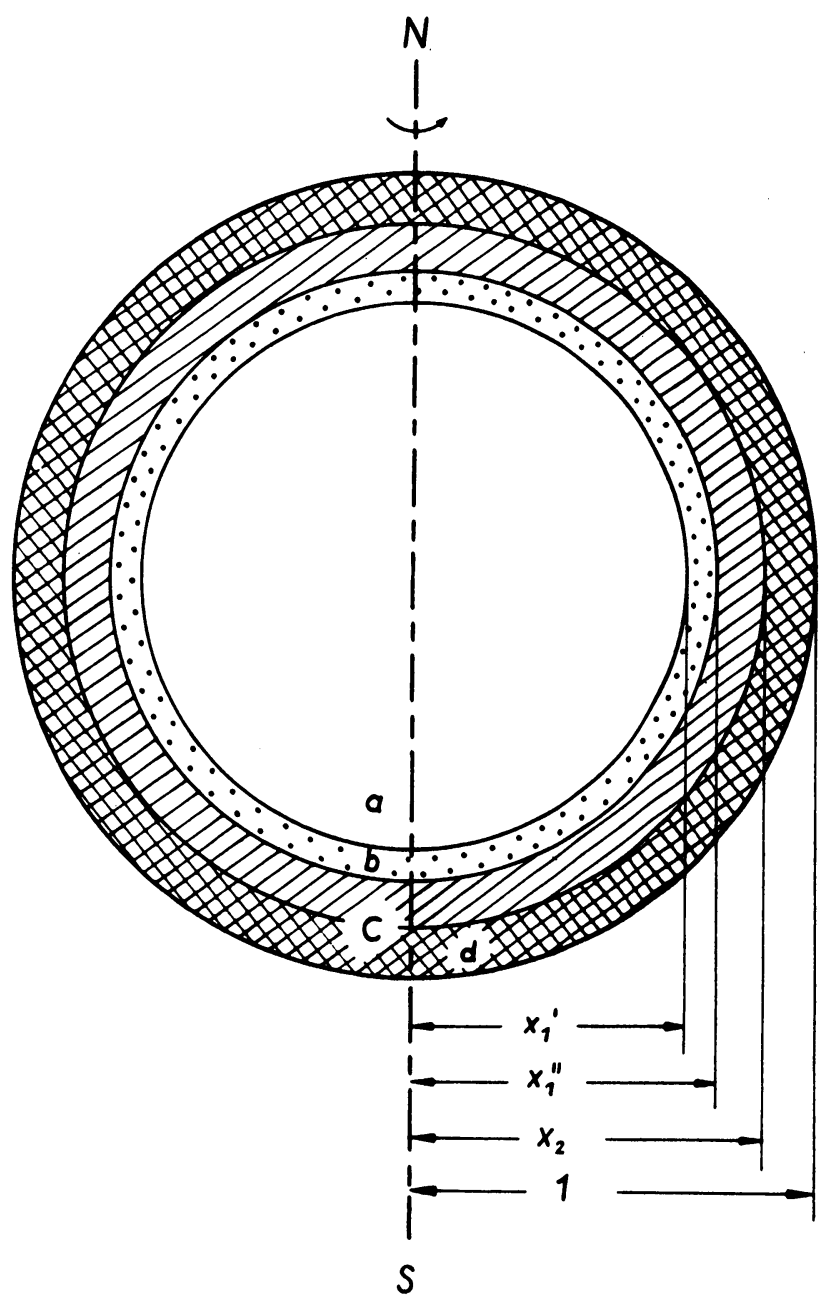

Fig. 5. Representation of the basic type of the investigated spherical alternating field dynamos. $a$ denotes a rigid rotating core and $c$ a spherical shell also rotating rigidly but with a lower angular velocity. $b$ denotes the transition shell and $d$ a shell with $\alpha$-effect. 
It may be remarked that the separation of the two layers must not be complete; there can occur an overlapping.

Surprisingly a butterfly diagram is obtained quite similar to the observed one taking into account an $r$ dependence of the angular velocity only. This result suggests that for the production of the toroidal field of the Sun the radial variation of the angular velocity is more important than the variation with latitude, as has been believed till now. An observation of Wilcox (1971) supports this conception. According to this observation the active regions show rigid rotation and do not take part in the differential rotation observed at the surface of the Sun.

It may be underlined that the alternating field dynamo presented here only works if the two induction mechanisms act on different layers. This is a definite statement concerning the structure of the Sun which would be of less importance if there were other alternative models. Therefore it is of interest to analyze the model of Leighton (1969). It seems that Leighton involved a certain singularity in his model. In Equation (4) of his paper a factor $\sin \theta$ is missed. Therefore a toroidal field for the production
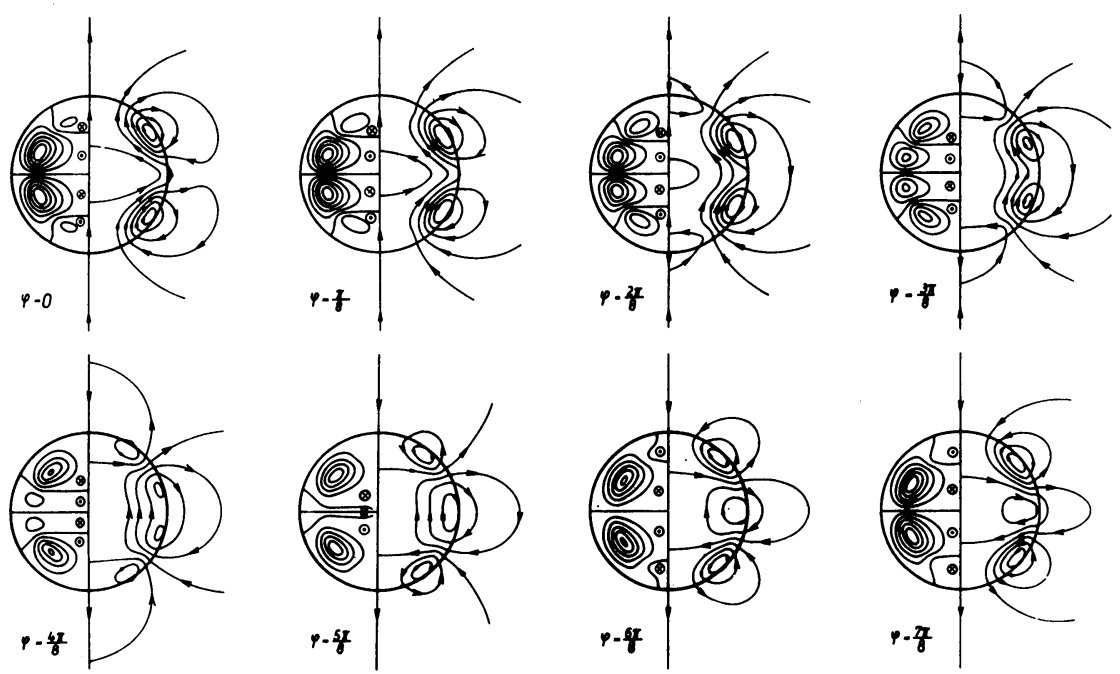

Fig. 6. Eight phase pictures of the field maintained by a calculated dynamo. The right half of every phase picture shows the field lines of the poloidal field whereas the left half shows the lines of constant field strength of the toroidal field. The phase $\Omega t=0$ corresponds to maximum toroidal field strength. The migration of the field towards the equator should be noted. Further attention may be drawn to the region of the phases where the dipole changes its sign (about $5 \pi / 8 \ldots 6 \pi / 8$ ). The field configuration is determined by the higher multipoles there.

of $B_{r}$ is assumed, which is singular at the poles of the Sun. At first this singularity is excluded by the assumption of the threshold field $B_{c}$, but the results show a dying oscillatory field. Therefore Leighton for a fraction of the $B_{r}$ field, takes this equation without the threshold condition. Now the oscillation is maintained but the singularity is included too. For this reason the Leighton model cannot be accepted as an alternative. 


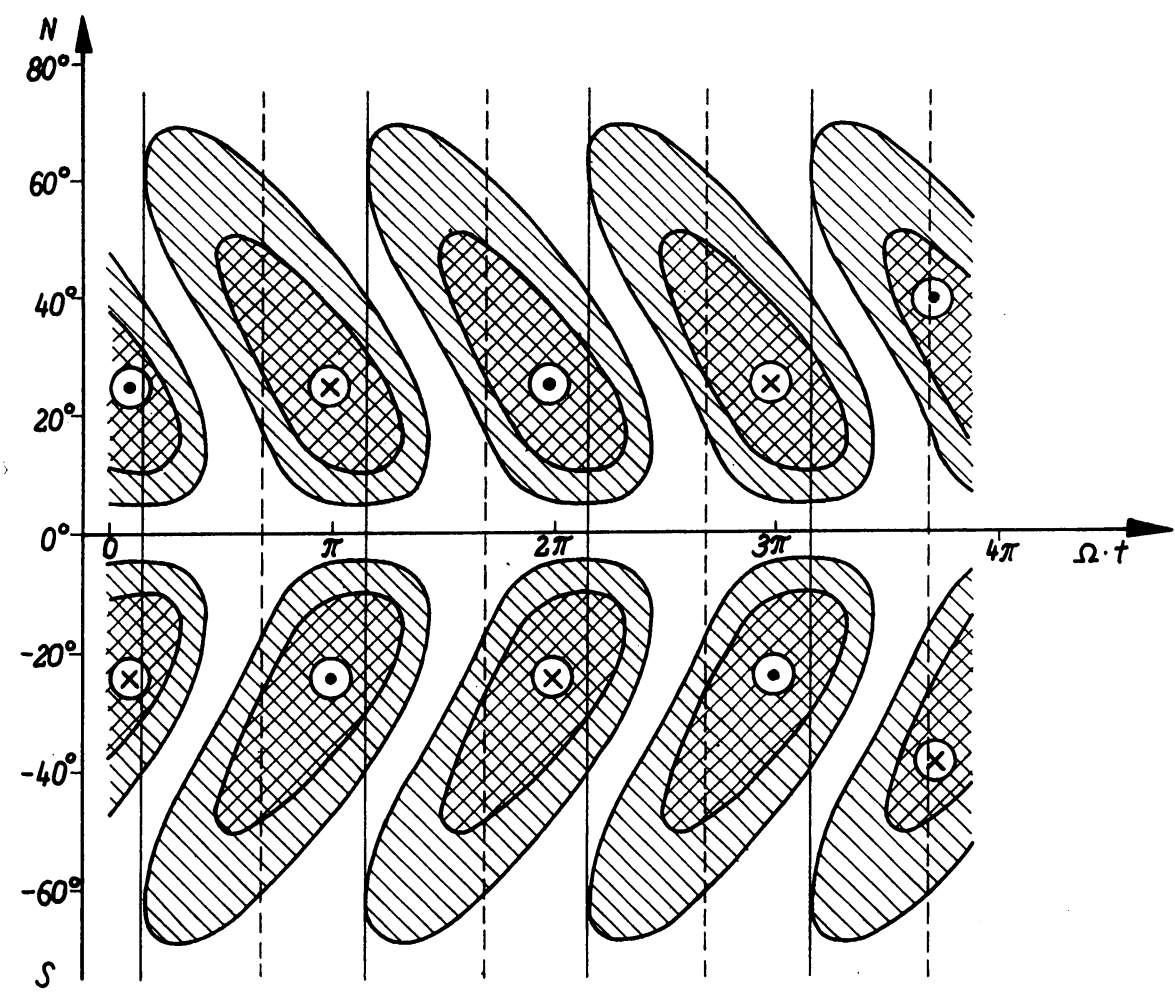

Fig. 7. Butterfly diagram derived from a calculated model. The typical observed behavior, i.e. the migration towards the equator, can also be noticed here. The dotted line denotes the phase where the dipole changes its sign.

\section{Conclusions}

We promised to furnish a dynamo satisfying the requirements (1)-(4). Concerning requirement (1) we have to remark that the $\alpha$-effect is a quite natural one appearing in turbulence in rotating systems as already mentioned. The second induction mechanism is provided by the differential rotation. There is no doubt about the fact that both effects will occur at the Sun, but their radial dependence is rather unknown. We can assume this dependence according to what is needed for having a dynamo because there is no completely developed theory. If a similarity appears between the calculated and the observed field the dynamo model can be considered as a probe giving information about the structure of the layers of the Sun which take part in the dynamo excitation. A first hint in this direction is the statement that there is a strong $r$ dependence of the angular velocity at deeper layers. This statement is supported by the observation of Wilcox (1971) already mentioned. More detailed information can be expected from the non-linear theory.

Requirement (2) is satisfied because of the linearity of the equations describing the dynamo. Requirement (3) needs no further discussion. 
Concerning requirement (4) it may be remarked that the field equations have eigen solutions which are symmetric in relation to the equatorial plane - the leading term for the poloidal field is a quadrupole - in addition there are antisymmetric solutions with a dipole as the leading term. A model will excite that field which makes the smallest pretensions to the induction effects, in other words, which has the smallest eigenvalue characterizing the pretension. The eigenvalue for the symmetric field was proved by calculations to be greater than that for the antisymmetric field. Hence the antisymmetric field will be excited.

As we have mentioned an accurate fitting of the models to the observations is only reasonable in the framework of a non-linear theory. For this purpose it is desirable to derive as much information as possible concerning the space-time behavior of the mean field. The butterfly diagram is one kind of evaluation of the observational data. Another one could be the determination of the dependence on time and latitude of the mean poloidal field at the surface of the Sun. A first step in this direction has been carried out by Stenflo (1971) with encouraging results.

\section{References}

Babcock, H.: 1961, Astrophys. J. 133, 572.

Fitremann, M. and Frisch, U.: 1969, Compt. Rend. 268, B705.

Krause, F.: 1968, Habilitationsschrift, Univ. of Jena.

Krause, F.: 1969, Proc. 4th Consult. Solar Phys. and Hydromagn., Sopot 1966, Acta Univ. Wratislaviensis No. 77, 157.

Krause, F.: 1969, Mber. Deut. Akad. Wiss. Berlin 11, 188.

Krause, F. and Steenbeck, M.: 1965, Proc. 3rd Consult. Solar Phys. and Hydromagn., Tatranska Lomnica 1964, Czech. Acad. Sci. Astron. Inst. Publ. 51, 36.

Leighton, R. B.: 1969, Astrophys. J. 156, 1.

Moffatt, H. K.: 1970, J. Fluid Mech. 41, 435.

Rädler, K.-H.: 1968, Z. Naturforsch. 23a, 1841, 1851.

Rädler, K.-H.: 1969, Mber. Deut. Akad. Wiss. 11, 194.

Steenbeck, M. and Krause, F.: 1966, Z. Naturforsch. 21a, 1285.

Steenbeck, M. and Krause, F.: 1968, Astron. Nachr. 291, 49, 271.

Steenbeck, M., Krause, F., and Rädler, K.-H.: 1966, Z. Naturforsch . 21a, 369.

Stenflo, J. O.: 1971, this volume, p. 714.

Weinstein, S. I.: 1970, J. Exp. Theor. Phys. 58, 153.

Wilcox, J. M.: 1971, this volume, p. 744.

\section{Discussion}

Gilman: I am concerned about the separation required by the two dynamo processes (differential rotation and $\alpha$-effect). It seems to me that the $\alpha$-effect due to swirl motion induced by the basic rotation may be just as strong, or stronger, near the bottom of the convection zone where you are also assuming the differential rotation effect to be strongest.

Krause: Apparently $\alpha$ rises at first with the depth inside the convection zone, but it is zero at the bottom. The wanted radial variation of the angular velocity $\omega$ may be given in the layer just under the convection zone where we expect a smooth transition of $\omega$ to the value inside the core.

Nagarajan: My question is about the prescription of the pseudo scaler stochastic parameter $\alpha$ which effectively linearizes the problem. This has to be dynamically determined from the fluctuation equation, unless you neglect all fluctuations below a certain length scale (magnetic field or velocity). I do not see how the leaving out of the Lorentz term is consistent. For smaller scales even a crude testing would show that $\overline{v \cdot \nabla v}$ and $\overline{j \times B}$ would be of the same order. Secondly, in this approximation 
of treating $\alpha$ 's as prescribed stochastic parameters, we run into the statistical difficulty about separation of mean and fluctuation time scales, which is rather difficult in turbulence.

Krause: $\alpha$ is determined by a certain disturbation method from the Navier-Stokes equations. If we include Lorentz forces, $\alpha$ is lowered by a term proportional to the square of the mean magnetic field. It should be added that the $\alpha$-effect was also experimentally proved by an experiment carried out in the Institute of Physics in Riga using liquid sodium. 